\title{
Développement Economique et Croissance des Risques *
}

\author{
par Orio Giarini **
}

\section{Introduction}

Depuis les années 1960, un même phénomène conditionne l'évolution économique générale, ainsi que la croissance de la fonction du risk management : la modification profonde du processus d'industrialisation et de l'impact économique de la science et de la technologie. D'autres phénomènes (culturels, politiques, institutionnels) conditionnent bien entendu aussi l'évolution économique, mais - à notre avis - ils ne sont pas déterminants au point de pouvoir, à eux seuls, et même savamment conjugés, renverser les tendances actuelles et nous ramener à une situation de croissance économique généralisée comme celle que nous avons expérimentée pendant un quart de siècle après la deuxième guerre mondiale, réduisant du même coup les problèmes que le risk management tente de résoudre.

La naissance et l'évolution de la fonction de risk management est donc liée à quelque chose de fondamental au niveau économique général, et aux nouvelles exigences de gestion de toutes les entreprises et institutions qui, dans la société, contribuent à la production des biens et des services qui déterminent le niveau de richesse et de bien-être.

\section{Les origines de la décroissance économique}

\subsection{Les rendements décroissants de la technologie}

Depuis 1973 et la « crise du pétrole », on a pu constater les effets économiques suivants :

- l'augmentation du prix du pétrole n'a pas permis de développer rapidement de nouvelles technologies qui compensent le fait que les forages ont lieu aujourd'hui

* Papier présenté à la Conférence Internationale de Risk Management, Monte-Carlo, 12-14 octobre 1981.

** Secrétaire Général de l'Association de Genève (Association Internationale pour l'Etude de l'Economie de l'Assurance) et Membre du Club de Rome. 
dans des parties du monde où le pétrole est plus difficilement accessible et donc plus cher à extraire que dans la plupart des pays du Moyen-Orient ;

- parallèlement, même si la presse a rendu les sources d'énergie dites «alternatives » très populaires (le nucléaire, les énergies renouvelables, les méthodes d'économie d'énergie), il est vite apparu que ces sources ne permettaient pas non plus de fournir de l'énergie bon marché et de nous ramener à la situation qui prévalait pendant les années soixante. Bien au contraire, les estimations des coûts de toutes les nouvelles formes de production d'énergie continuent à grimper d'une façon constante par rapport aux espoirs exprimés dans les études technico-économiques qui avaient été faites, pour toutes ces sources, depuis plusieurs décennies;

- en outre, si l'on a constaté d'une part que l'énergie est devenue de plus en plus chère (en termes économiques plus précis, on dirait que son "prix relatif " a augmenté), on a pu aussi vérifier que l'ensemble du monde industriel n'a pas pu se reconvertir assez rapidement pour réduire sa consommation d'énergie de façon substantielle. Il aurait été en effet concevable, selon certains, qu'au moins de ce côté-là, la technologie puisse réagir assez rapidement pour que la consommation soit réduite de façon décisive. Les progrès qu'on a pu constater ont été dans l'ensemble bien en-deçà de ces espoirs.

Ces trois considérations montrent bien qu'en 1973, un événement de nature politique est devenu le grand révélateur d'un processus qui avait déjà ses racines dans les années soixante, et que l'on peut définir économiquement comme une augmentation des rigidités de l'offre. Et ceci à cause d'un phénomène précis, celui des rendements décroissants de la technologie.

Nous ne nions nullement par là les progrès encore considérables que la technologie permet d'accomplir dans divers secteurs économiques, mais nous soulignons le fait que ces progrès technologiques n'arrivent pas à compenser toute une série de contraintes économiques (augmentation de la population mondiale, raréfaction des ressources, coût croissant de la protection de l'environnement, augmentation de la vulnérabilité des systèmes économiques industriels), comme cela fut le cas pendant deux siècles de révolution industrielle. Il est indéniable, par exemple, que dans le secteur de l'électronique on assiste à des progrès qui, même s'ils sont parfois surestimés, n'en représentent pas moins une amélioration économique dans l'utilisation des ressources. Toutefois, ce phénomène n'est pas suffisamment généralisé pour compenser, à lui seul, l'augmentation des contraintes qui se traduit par une croissance de l'inflation et du chômage.

Nous avons traité ailleurs le problème des rendements décroissants de la technologie (voir Giarini et Loubergé [1979]). Nous voudrions ici en rappeler quelques points essentiels :

a) La technologie a été la principale source des économies d'échelle. Les gains de productivité acquis tout au long de la révolution industrielle étaient énormes, mais c'est seulement maintenant que l'on examine d'une manière plus critique les avantages d'une augmentation de l'échelle d'exploitation. En effet, certains signes montrent que l'augmentation du rendement due à une augmentation de l'échelle commence à diminuer et même à produire des résultats négatifs dans l'absolu ; en 
outre, la concentration de la production se traduit par des coûts indirects (pour le stockage, le contrôle de la qualité, la coordination aux différents stades de la production, la vulnérabilité vis-à-vis de faibles défaillances, etc.) tellement élevés qu'au-delà d'un certain seuil, le coût global dépasse souvent le total des avantages correspondants.

C'est dans ce domaine que les risques et la vulnérabilité deviennent de plus en plus des risques "purs », assurables.

b) La spécialisation a aussi été un facteur fondamental de progrès, mais une spécialisation poussée à outrance produit des systèmes de plus en plus interdépendants et de plus en plus vulnérables, comme le démontre l'augmentation de toutes sortes de risques indirects. En outre, la spécialisation réduit souvent les capacités d'adaptation des machines ou des installations aux conditions changeantes du marché, et impose des règles d'entretien et de réparation beaucoup plus sévères, qui risquent d'être difficiles à appliquer à chaque changement de situation. Le gain que produit la spécialisation peut être neutralisé en totalité ou en partie, voire annulé, par le manque de flexibilité qui en résulte.

c) La technologie a fait de grands progrès dans le domaine de la fiabilité. Le revers de la médaille est que les variations mineures et les petits accidents produisent souvent des dommages plus importants, même si leur fréquence est plus faible.

d) Il ne fait pas de doute que la technologie moderne a fait beaucoup pour améliorer non seulement la quantité, mais aussi la qualité d'un grand nombre de produits. Mais nous sommes en train de découvrir que l'amélioration de la qualité d'un produit pour une tâche particulière peut augmenter le problème de recyclage quand on jette ce produit. D'après Alfred Marshall, l'environnement humain et économique est beaucoup plus proche d'un processus biologique que d'un processus mécanique. Toute amélioration dans un sens introduit un déséquilibre dans l'autre. Cette leçon, nous l'avons apprise dans le cadre du problème de la lutte contre la pollution.

e) Au cours des dernières décennies, grâce à l'organisation professionnelle de la recherche, des possibilités de nouvelles technologies ont été étudiées et testées presque en temps réel. Le retard dû à un manque de compréhension des technologies disponibles ou adaptables est presque ramené à zéro. En fait, les retards dans l'évolution technologique sont aujourd'hui de plus en plus de nature technique. Plus la technologie est avancée et complexe, plus il faut de temps pour la rendre convenablement et économiquement viable. Tandis que jusqu'au début de ce siècle, le temps nécessaire pour passer de l'invention à l'utilisation des nouvelles technologies était très long pour des raisons essentiellement culturelles : on devait apprendre que les découvertes et les inventions nouvelles des ingénieurs pouvaient vraiment aboutir à une utilisation pratique.

f) En outre, la technologie moderne dépend souvent de la recherche fondamentale qui supporte la contrainte de délais encore plus longs (de l'ordre d'une ou plusieurs décennies). Nous sommes entrés dans une phase de délais techniques croissants, en contraste avec le mythe de «l'accélération technologique», qui est un fait culturel et qui cache une réalité technique aux caractéristiques opposées. 


\subsection{La rigidité de l'offre, cause principale de la décélération de la croissance économique}

C'est à cause des rendements décroissants de la technologie que nous assistons donc depuis huit ans à un accroissement de la rigidité de l'offre, c'est-à-dire des possibilités réelles de développer l'investissement afin de retrouver une situation d'équilibre qui puisse éliminer l'inflation et absorber le chômage.

Il est frappant de souligner que ce manque de dynamique de l'activité de l'investissement a été observé dans les situations les plus variées. Mais, généralement, l'analyse économique s'est, jusqu'ici, bornée surtout à examiner le problème du côté de la demande (qu'il s'agisse des économistes keynesiens et même, pour une large part, des monétaristes). De notre point de vue, ils insistent encore à regarder donc du mauvais côté. Sur le plan de la politique économique, certains gouvernements ont imputé le manque de dynamisme de l'investissement à l'excès d'interventions de l'Etat, tandis que d'autres ont imputé ce même manque à une intervention insuffisante de l'Etat. Les deux analyses, en réalité, se rejoignent profondément, parce qu'elles présupposent une situation dans laquelle l'offre, c'est-à-dire l'investissement, serait "naturellement » élastique et susceptible d'être relancée à volonté à condition tout simplement de développer un environnement institutionnel convenable.

Ce n'est pas du tout notre avis et nous aimerions insister ici encore une fois sur le fait que le problème essentiel qui est apparu depuis 1973 est justement celui d'une situation de plus en plus rigide du côté de l'offre, qui s'est d'ailleurs traduite par une diminution assez constante des taux de profit, et qu'en définitive les responsables de la recherche et de l'investissement de la plupart des sociétés du monde industriel connaissent assez bien.

Pour toute une série de raisons, lorsque la crise a éclaté en 1973, on a préféré espérer qu'il s'agissait d'une crise conjoncturelle, c'est-à-dire que, après quelques années d'ajustement, on serait revenu à la croissance «normale ». C'est ainsi que, par exemple, autour des années 1975 , les gouvernements ont souvent surtout essayé de mener une politique de transition, en acceptant de plus en plus d'accroître le déficit budgétaire de l'Etat et d'une façon générale du secteur public. Aujourd'hui, la situation assez difficile et nerveuse, sur le plan monétaire et financier, montre à quel point les mesures prises à la suite du premier choc du pétrole sont aujourd'hui en grande partie responsables de la généralisation de la crise économique sous la forme d'instabilité monétaire et financière. La principale contradiction économique est la suivante : une situation de rendements décroissants de la technologie a pour effet de diminuer la demande de capital, ce qui s'est répercuté jusqu'il y a un ou deux ans dans la tendance des taux d'intérêt à se situer en dessous de zéro, en termes réels, surtout si l'on tient compte de la fiscalité. Mais, en même temps, l'accroissement des déficits budgétaires et publics, considéré à l'origine comme un moyen transitoire pour surmonter une crise conjoncturelle, fait diverger de plus en plus les flux financiers vers des utilisations non productives, et, étant donné l'énormité de ces déficits, pousse les taux d'intérêts à des niveaux extrêmement élevés et qui ne trouvent aucune justification si on regarde la rentabilité réelle du capital investi dans l'activité productive.

Voilà donc, dans notre interprétation, l'analyse que l'on peut faire actuellement de la situation économique et l'explication des phénomènes apparemment irrationnels 
auxquels nous assistons. A partir de là, il convient de mener une politique économique consciente des limites réelles de l'offre et tirer toutes les conséquences qui s'imposent pour que l'ajustement ne se fasse pas dans les pires conditions. Dans le dernier rapport au Club de Rome (Giarini [1981]), nous avons essayé d'esquisser quelques propositions constructives à cet égard.

\section{La croissance de la vulnérabilité}

Considérons maintenant la façon dont les causes profondes de l'évolution économique que nous avons vécue depuis 1973 (mais qui était déjà à l'œuvre pendant les années soixante) est également directement liée à la croissance de la fonction de risk management dans les entreprises.

L'un des effets majeurs des rendements décroissants de la technologie est d'augmenter la croissance de la vulnérabilité des systèmes économiques à plusieurs niveaux. C'est ainsi que se sont créés les prémisses d'une interpénétration de plus en plus profonde entre les risques d'entrepreneurs et les risques purs.

Commençons par distinguer du point de vue économique ces deux catégories de risques :

a) Les risques d'entrepreneurs : ils concernent les décisions prises en matière d'investissement, de production, de recherche et de marketing. Ils engagent la volonté et la responsabilité de celui qui les prend (une personne ou une institution) motivé par l'espoir d'atteindre un objectif (un avantage, un profit) donné.

b) Les risques dits purs: ils concernent les événements imprévisibles et qui peuvent interrompre, faire diverger et même annihiler toute activité ; ils échappent en principe à la volonté consciente des hommes (incendie accidentel d'une maison ou d'une fabrique, explosion accidentelle, tremblement de terre, etc.).

En économie, on a eu l'habitude, pendant la période classique de la révolution industrielle, de dissocier ces deux types de risques. En effet, seul le premier a vraiment attiré l'attention des économistes, le deuxième étant assimilé très souvent à un simple coût du hasard, que l'on pouvait contrôler par l'achat d'une police d'assurance.

En réalité, un des effets de la complexité croissante de la société industrielle et l'une de ses principales caractéristiques a été que, de toute évidence, durant ces dernières décennies, les risques purs et les risques d'entrepreneurs se conditionnent de plus en plus étroitement les uns les autres.

Il suffit d'ailleurs de comparer les décisions stratégiques prises par le Conseil d'Administration d'une entreprise importante aujourd'hui, à celles que l'on prenait au début du siècle pour remarquer que les questions concernant les risques purs étaient toujours (sauf peut-être dans les société fabriquant des explosifs), du ressort d'un employé de niveau secondaire. Aujourd'hui, les questions touchant la responsabilitéproduits ou l'ensemble des vulnérabilités au niveau de production et de distribution sont au cœur même des activités d'investissement qu'elles conditionnent. 
Nous touchons là du doigt l'une des conséquences du processus de rendements décroissants de la technologie. Je le répète : nous sommes bien d'accord pour dire que la technologie moderne est devenue très efficace. Pourtant, paradoxalement, elle est également devenue, sous bien des aspects, de plus en plus vulnérable. Et ceci directement autant qu'indirectement. Par exemple :

- Le coût total des incendies en Europe est actuellement de l'ordre de $1 \%$ du produit national, et présente une croissance plus que proportionnelle. (La part prise en charge par les assurances est de l'ordre du tiers du total.)

- Le coût économique de la criminalité est plusieurs fois supérieur à celui des incendies. La raison en est non pas tant une augmentation du nombre des délits et des crimes, que la vulnérabilité des systèmes vis-à-vis des activités criminelles. On peut dire la même chose à propos des catastrophes naturelles: les dommages provoqués par des tremblements de terre et des inondations sont plus importants à l'heure actuelle, mais pas nécessairement parce que les causes des sinistres sont différentes, mais parce qu'ils affectent surtout un système plus vulnérable.

- D'après une étude de l'Association de Genève en Europe, et de Skandia aux EtatsUnis, le coût économique total des dommages indirects " produits » par l'utilisation de systèmes informatisés pourrait, dans une dizaine d'années, atteindre près de 6 milliards de dollars par an. Il faut évidemment comparer ce chiffre aux avantages, qui seront probablement plus élevés; il n'en reste pas moins qu'il y a là de la place pour une gestion convenable du risque, c'est-à-dire pour le « Risk Management ».

- La plateforme de Statfjord, dans la mer du Nord, a coûté plus d'un milliard de dollars, sans tenir compte de l'infrastructure et du coût de l'enlèvement de la plateforme après utilisation. Il ne sera pas facile de suivre ces niveaux d'investissement, tant du point de vue financier que du point de vue de la couverture d'assurance. La plateforme de Statfjord est un exemple d'une tendance mettant en jeu un nombre de plus en plus grand d'activités industrielles et des services de plus en plus variés.

Il est vrai aussi que, si les accidents sont souvent de moins en moins nombreux, ils sont de plus en plus graves jusqu'au point qu'un seul accident peut constituer une catastrophe. Le fait que les catastrophes accidentelles soient rares, mais qu'elles puissent se produire aujourd'hui ou seulement dans cinquante ans, est un facteur supplémentaire d'incertitude.

A propos du coût des accidents et de leur dimension, il faut aussi mettre bien au clair un problème : on dit souvent que cent accidents dont chacun coûterait $1000 \$$ sont l'équivalent d'un seul accident qui, lui, coûterait $100.000 \$$. En réalité, les deux situations sont profondément différentes. Elles le sont d'autant plus si l'accident est rare et spécifique, et de nature catastrophique (c'est-à-dire de nature à détruire complètement le système dans lequel il se produit). En effet, lorsque la vulnérabilité est petite, même si les accidents se produisent souvent, ils sont facilement réparables et le système peut continuer à exister. Lorsque les risques sont très élevés, mais que leur fréquence est très rare, il y aura un grand élément d'incertitude et on peut finir par dépasser le seuil technique au-delà duquel un risque n'est plus normalement assurable. Ce concept n'a rien à faire avec des problèmes contingents de politique dans le domaine des services et des assurances. Il découle simplement du fait que, lorsqu'il n'y a pas d'univers 
statistique auquel se référer, on est dans l'impossibilité d'utiliser l'assurance qui, à un niveau de vulnérabilité moindre, est un instrument essentiel de contrôle des risques purs.

Une mesure importante de la dimension économique optimale dans toutes les activités économiques devrait justement être celle de considérer leur assurabilité technique. Du moins dans une société pluraliste.

On peut dépasser, et on dépasse très souvent, le seuil de cette assurabilité technique, pour des raisons qui touchent par exemple à la sécurité nationale. Dans ce domaine, on sort aussi traditionnellement de l'analyse économique, c'est-à-dire d'une analyse qui comptabilise et compare les coûts à supporter pour obtenir des objectifs spécifiques. Ceci dit, il faut insister sur l'importance qu'il y aurait en analyse économique à évaluer l'optimum des dimensions avec les niveaux d'assurabilité de toutes les activités économiques.

Il serait donc extrêmement fructueux de lier l'analyse actuarielle définissant les paramètres de fréquences et d'amplitudes de toutes les situations de vulnérabilités aux analyses d'économie d'échelle. Voilà un domaine où la contribution conjointe des économistes et des actuaires et des risk managers pourrait être d'une très grande importance.

Mais il y a un autre aspect dont il faut tenir compte si on admet l'hypothèse selon laquelle la technologie moderne a eu comme effet d'accentuer très souvent directement ou indirectement les niveaux absolus de vulnérabilité.

A partir du moment où les niveaux d'assurabilité sont de plus en plus souvent dépassés, le recours normal et habituel est celui de l'Etat. Voilà donc que, par le biais d'une augmentation de la vulnérabilité, nous pouvons détecter une tendance, que l'on pourrait appeler technique, à l'étatisation des activités économiques, qui s'opère en dehors de tout schéma politique préconçu. Voilà un point où l'économie et le sociopolitique se rejoignent, et qu'il faut faire très attention d'étudier dans toutes ses implications.

Il y a bel et bien accumulation de risques purs et de risques d'entrepreneurs lorsque, par exemple, la vulnérabilité d'un certain nombre de projets est telle que l'investissement même devient de plus en plus incertain. C'est alors que l'on trouve dans des revues comme la "Harvard Business Review» des considérations de ce type: - L'obstacle principal à l'obtention d'un capital suffisant pour des investissements nouveaux et considérables vient de la nécessité de développer de nouvelles ressources et une nouvelle technologie à un rythme trop risqué pour être accepté par les seuls investisseurs privés. La solution consiste à utiliser la capacité des pouvoirs publics d'assurer ou de couvrir la plus grande partie de ces risques extraordinaires. „ (Lindsay [1978]). Jusqu'où peuvent mener de telles ouvertures? C'est toute la question !

\section{L'interpénétration entre risque d'entreprise et risque pur}

C'est donc pour des raisons profondes liées au changement des caractéristiques mêmes de la révolution industrielle, que nous constatons aujourd'hui de plus en plus une intégration de la fonction de risk management dans la fonction de gestion de 
risque de l'entreprise. Ce qui ne veut pas dire, naturellement, que toute activité de risk management de l'entreprise possède les caractéristiques d'une activité d'importance stratégique. Mais cela veut bien dire que la subdivision entre risque pur et risque d'entrepreneur ne peut plus être verticale, que les deux s'intègrent horizontalement là où la gestion de la vulnérabilité devient un problème fondamental dans la gestion de l'entreprise tout court. Le schéma suivant permet de visualiser cette intégration.

Parler, en outre, de fonction de risk management ne veut pas dire parler nécessairement des risk managers : nous entrons là dans un domaine d'analyse qui a déjà été traité par ailleurs, notamment par M. Haller [1976] [1978].

Tableau 1: L'interdépendance entre risque pur et risque d'entrepreneur

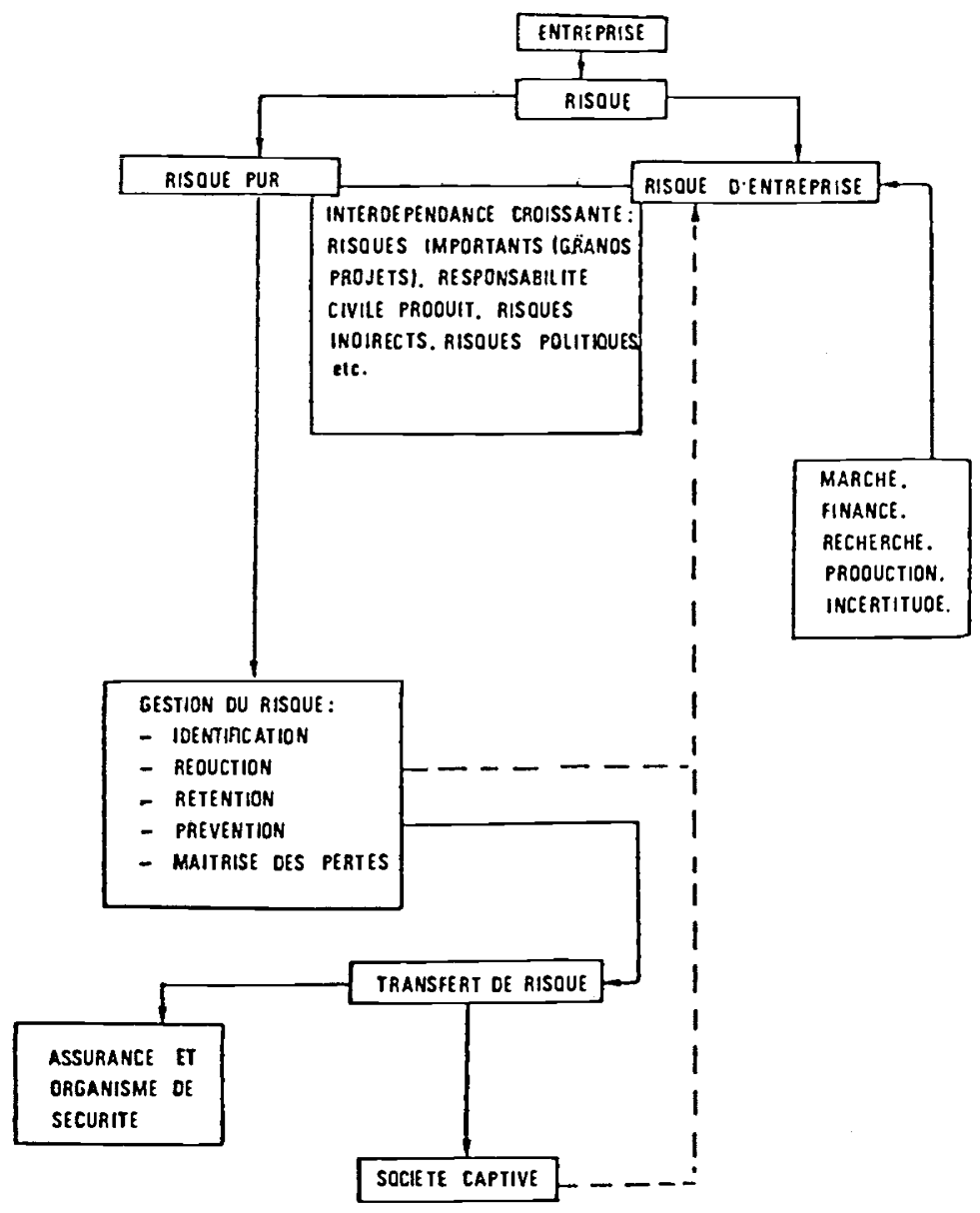




\section{L'importance croissante de l'assurance}

L'évolution de la nature du risque dans les sociétés industrielles (ou plutôt désormais post-industrielles) a non seulement eu comme conséquence de «produire » la notion de risk management, mais d'une façon tout aussi concrète, d'accélérer l'importance économique globale de l'assurance. Cette dernière représente en effet un outil important dans le cadre du risk management et une récente étude de SIGMA [1981] met en évidence comment l'assurance est devenue, depuis trente ans, de plus en plus importante par rapport aux autres secteurs de l'économie et que sa croissance relative a même continué à un taux élevé pendant les « années de crise ».

Tableau 2: Volume mondial des primes de 1950 à 1979

(affaires directes)

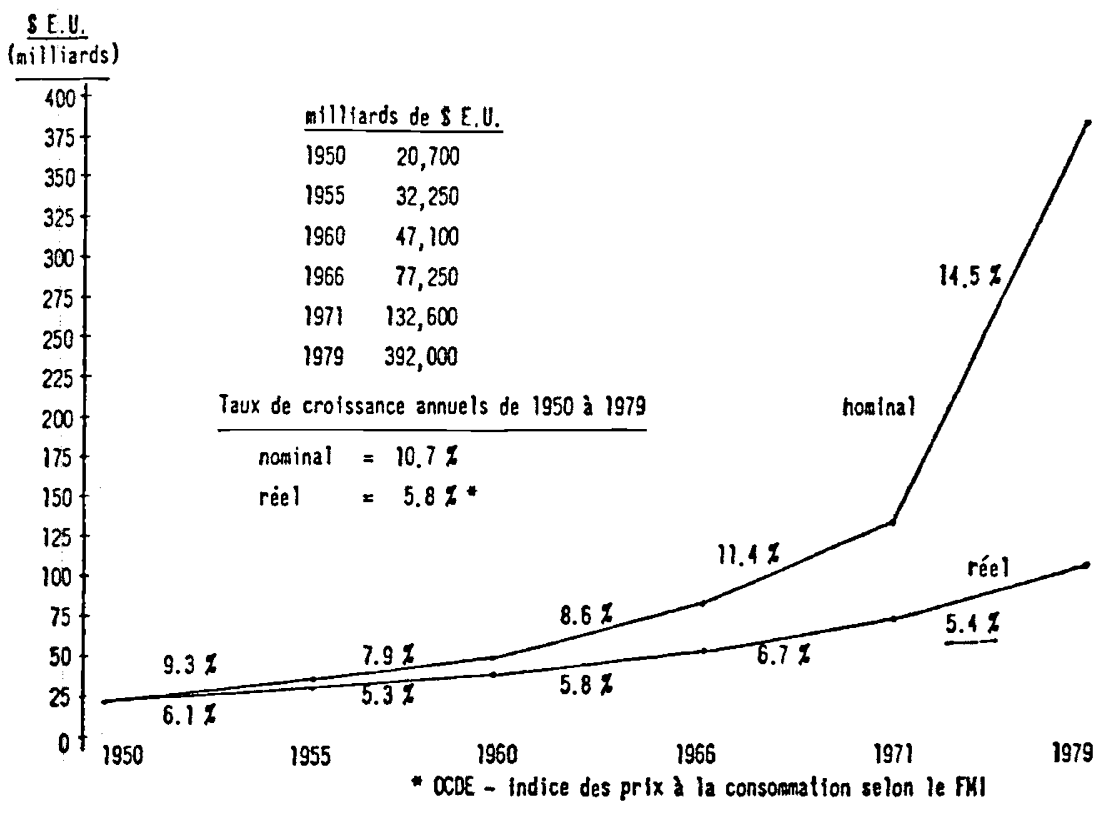

Source : SIGMA [1981] 
En ce qui concerne le développement de l'assurance par grande région économique, il est intéressant d'étudier le tableau suivant :

Tableau 3: Assurance mondiale et économie mondiale de 1950 à 1979

$$
\text { Primes en pourcentage du PNB }
$$

\begin{tabular}{|c|c|c|c|c|c|c|}
\hline & 1950 & 1955 & 1960 & 1966 & 1971 & 1979 \\
\hline$-\mathrm{OCDE}$ & 4,24 & 4,46 & 4,86 & 4,99 & 5,32 & 5,38 \\
\hline - Amérique du Nord & 5,16 & 5,57 & 6,15 & 6,23 & 6,79 & 7,06 \\
\hline - Europe occidentale & 2,73 & 2,76 & 3,10 & 3,46 & 3,83 & 4,14 \\
\hline dont Marché Commun & 2,67 & 2,88 & 3,27 & 3,71 & 4,09 & 4,43 \\
\hline - Reste de l'OCDE & 1,65 & 2,55 & 3,05 & 3,83 & 4,27 & 4,94 \\
\hline - Reste du monde & 0,61 & 0,69 & 0,83 & 0,97 & 1,20 & 1,53 \\
\hline Monde & 3,61 & 3,87 & 4,24 & 4,40 & 4,71 & 4,78 \\
\hline
\end{tabular}

(chiffres en partie estimés)

Source : SIGMA [1981].

Dans son résumé, SIGMA tire les conclusions suivantes :

«-Depuis 1950, le volume mondial des primes s'est développé de façon favorable, malgré des difficultés structurelles en partie importantes et des contrecoups conjoncturels plus ou moins marqués.

- Pendant toute la période sous revue, on peut pratiquement observer une croissance réelle constante.

- Par rapport à l'économie mondiale, l'économie de l'assurance a connu une croissance surélastique.

- De même, au niveau régional, on peut déceler une croissance plus forte des marchés de l'assurance par rapport à l'économie.

- Au niveau de cette comparaison, le marché nord-américain de l'assurance se trouve, avec une relation primes/PNB de $7,06 \%$ en 1979 , de loin en première position par rapport aux autres régions.

- Les autres régions (Europe occidentale, Marché commun, « reste de l'OCDE ») se tenaient relativement de près avec des valeurs comparatives de $4,14 \%$ d 
$4,94 \%$ en 1979. Leurs développements respectifs devraient être à peu près comparables.

- De même, les pays compris dans le "reste du monde » accusent, selon la relation primes/PNB, un développement très favorable. La valeur comparative afférente a passé de 0,61\% en 1950 a plus de 1,5\% en 1979. »

Nous voudrions ajouter à l'analyse de SIGMA un simple commentaire de nature économique, qui est le suivant :

Les économistes traditionnels ont toujours avancé l'idée que la «consommation » d'assurances était la conséquence d'un niveau d'économie assez élevé : on n'achèterait de l'assurance qu'à partir du moment où un certain nombre de besoins fondamentaux sont satisfaits : il s'agit de la « loi d'Engel ». En réalité, cette « loi » présente les mêmes inconvénients de la séparation nette entre risque pur et risque d'entrepreneur.

En effet, surtout depuis les dix dernières années, on ne peut pas dire que l'augmentation de la consommation d'assurances est due essentiellement à une augmentation « subjective " de la demande : d'une façon générale, en période de crise - même de crise relative - c'est sur l'assurance qu'on essayerait de dépenser le moins. Mais, le besoin d'assurances augmente plus que proportionnellement justement à cause du fait que la demande de couverture a un caractère objectif: elle est la conséquence d'une situation de rendements décroissants de la technologie.

D'ailleurs, cette situation de «succès » ou de croissance pour l'assurance et pour le risk management ne va pas sans inconvénients : il y a de gros problèmes d'adaptation à surmonter, qui ne sont pas toujours faciles ni sans douleur.

Mais c'est pour les mêmes raisons économiques fondamentales que l'activité de l'assurance est en train de devenir un point de repère important et très intéressant pour l'analyse économique, ainsi qu'une activité essentielle pour la compréhension de l'évolution économique elle-même.

\section{Conclusion : vers une société de services (la société post-industrielle)}

Pour conclure, nous aimerions proposer ici encore un certain nombre d'idées et d'hypothèses.

La première est que tout ce que nous venons de décrire est une façon de définir les caractéristiques de la société de services (ou société post-industrielle) qui est en train de succéder à la société industrielle traditionnelle. Nous sommes déjà passablement avancés dans cette direction et nous avons traité ce sujet plus en détail ailleurs (voir Giarini [1981]).

Nous ne reprendrons ici qu'un tableau récapitulatif des faits essentiels qui ont caractérisé la révolution industrielle et qui sont en même temps ceux qui commandent sa transformation. On peut remarquer dans ce tableau, qui couvre environ 250 ans, l'importance stratégique de la notion de risk management. 


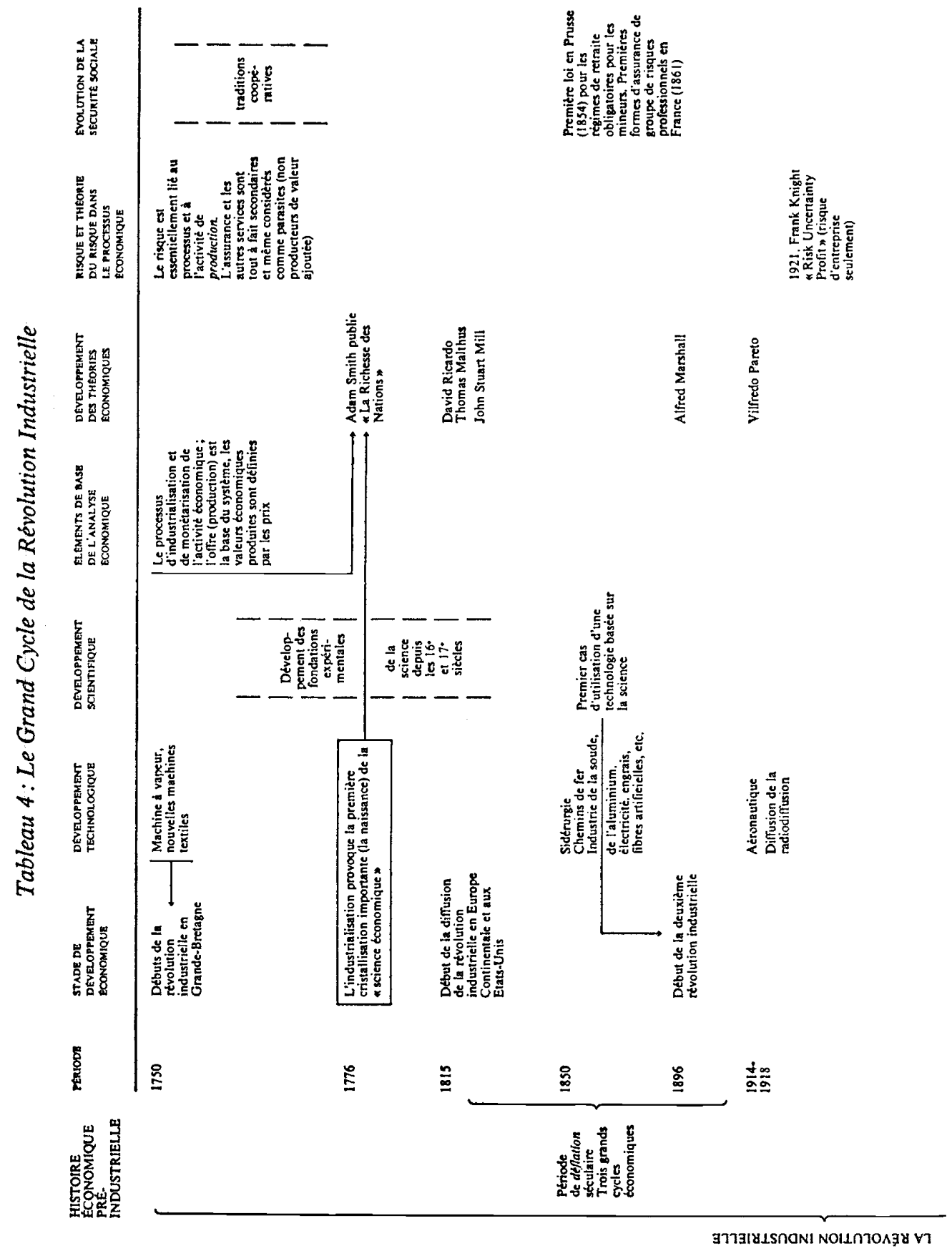




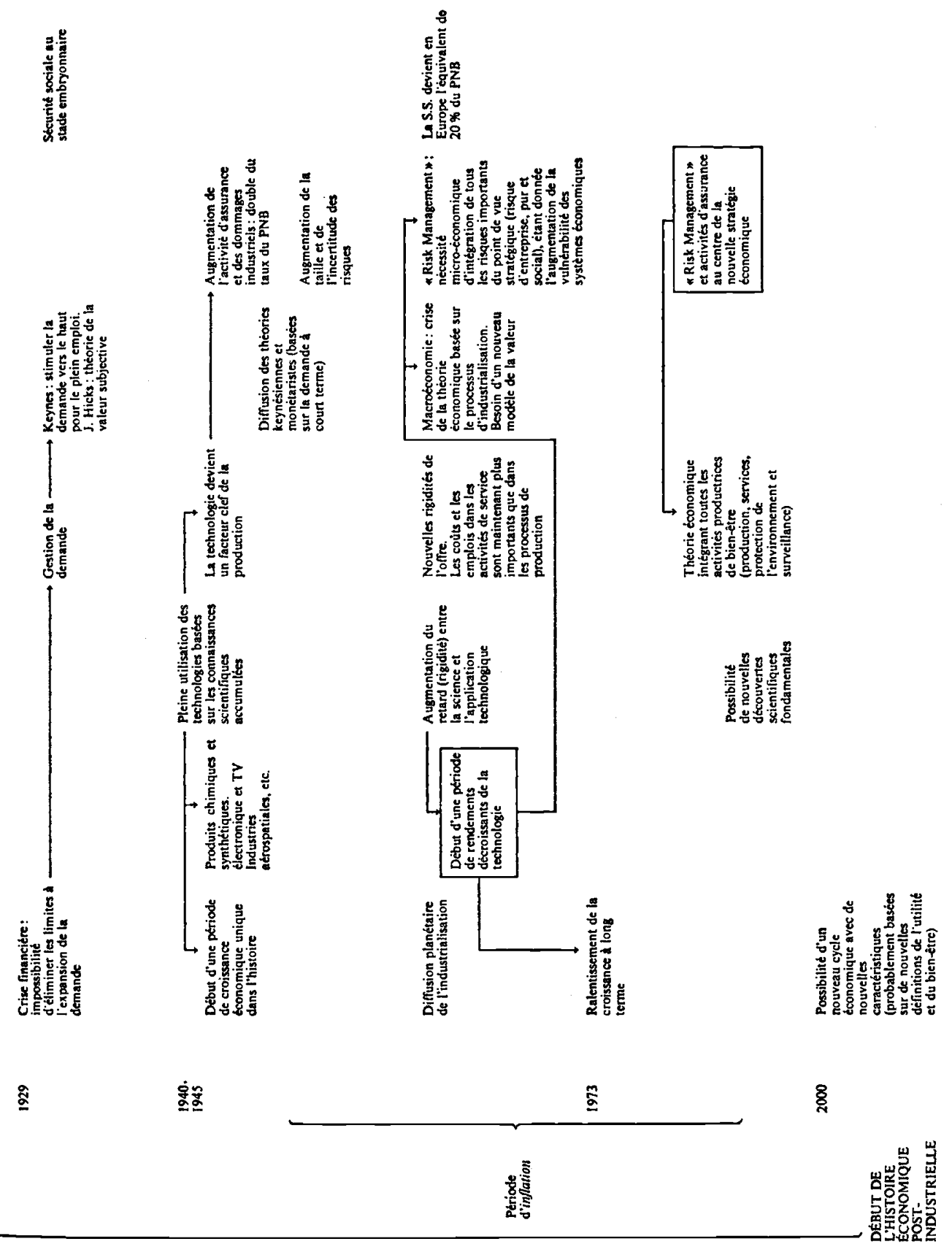


Dans cette société post-industrielle et de services, il se pose aussi d'autres problèmes importants comme celui de l'intégration des activités économiques qualitatives et en particulier de la * valeur» des services (voir Giarini [1981 bis]).

C'est dans ce cadre que la fonction de risk management prend tout son relief et se révèle pour ce qu'elle est vraiment : une fonction économique de plus en plus importante en vue de l'organisation des ressources humaines et matérielles pour produire dans les meilleures conditions la richesse et le bien-être des hommes et des femmes.

\section{REFERENCES}

GIARINI, O. [1981] : Dialogue sur la Richesse et le Bien-Etre, Economica, Paris.

GIARINI, O. [1981 bis] : «Développement économique, théorie économique et risques», Reflets et Perspectives de la Vie Economique, 20 (1/1981), 5-18.

GIARINI, O., et LOUBERGE, H. [1979] : La Civilisation Technicienne à la Dérive: Essai sur les Rendements Décroissants de la Technologie, Dunod, Paris.

HALLER, M. [1976] : "Les objectifs du risk management», The Geneva Papers on Risk and Insurance, 2 (août 1976), 12-26.

HALLER, M. [1978] : « New dimensions of risks: Consequences for Management », The Geneva Papers on Risk and Insurance, 7 (January 1978), 3-15.

LINDSAY, F. [1978] : "Financing high cost, high risk energy development 》, Harvard Business Review, 56 (November-December 1978).

SIGMA [1981] : "Croissance et structure de l'assurance dans le monde », Etudes Economiques de la Compagnie Suisse de Réassurances, juillet-août 1981. 